\title{
Caracterización de pequeños sistemas de producción de Vigna unguiculata en tres Municipios del Estado Portuguesa, Venezuela
}

\section{Characterization of small production systems of Vigna unguiculata in three Municipalities of Portuguesa State, Venezuela}

Danny Villegas ${ }^{1}$., Yary Pérez ${ }^{2}$., Salli Villegas ${ }^{3}$. y Manuel E Milla P ${ }^{4}$

\section{RESUMEN}

En este trabajo se presenta una caracterización para pequeños sistemas de producción de Vigna unguiculata "frijol" en los municipios Guanare, Papelón y San Genaro del estado Portuguesa con el objeto de interpretar y/o clasificar la producción. Fueron seleccionadas 43 fincas, utilizando información técnica, socioeconómica, agroecológica e institucional de los registros de la asociación de productores (ASOGUANARE S.A). Se evaluaron 17 variables, de las cuales 8 fueron descartadas por no reportar variabilidad. Sobre la matriz de datos conformada por nueve variables se aplicó un análisis de componentes principales (ACP) y una descripción estadística. De esta manera, el ACP permitió identificar seis factores que están relacionados con la producción de frijol los cuales son: por dimensión o tamaño (superficie total, superficie sembrada, superficie cosechada), ubicación geográfica o localidad, limitaciones por equipo y maquinaria, por cosecha y por mano de obra. Finalmente, se logró clasificar las 43 fincas en tres grupos discriminados principalmente por la dimensión o tamaño. Así mismo se observó que el grupo A reportó el rendimiento más alto $(630 \mathrm{Kg} / \mathrm{ha})$, lo que sugiere un grupo caracterizado por productores que se dedican principalmente al cultivo de frijol.

Palabras clave: análisis multivariado, factores, fincas, leguminosas.

\begin{abstract}
This paper presents the characterization of small production systems of Vigna unguiculata "beans" in the municipalities Guanare, Papelón and San Genaro Portuguese state in order to interpret and / or classify production. 43 farms were selected, using technical, socioeconomic, agroecological and institutional information from the producer association records (ASOGUANARE S.A). 17 variables were evaluated, of which 8 were discarded for not reporting variability. A principal component analysis (ACP) and a statistical description were applied to the data matrix consisting of nine variables. In this way, the ACP allowed to identify six factors that are related to bean production which are: by dimension or size (total area, area planted, area harvested), geographical location or location, limitations by equipment and machinery, by harvest and for labor. Finally, it was possible to classify the 43 farms in three groups discriminated mainly by size or size. Likewise, it was observed that group A reported the highest yield $(630 \mathrm{Kg} / \mathrm{ha})$, which suggests a group characterized by producers who are mainly engaged in bean cultivation.
\end{abstract}

Keywords: multivariate analysis, factors, farms, legumes.

\footnotetext{
${ }^{1}$ Universidad Nacional Experimental de los Llanos Occidentales "Ezequiel Zamora”, Guanaré, Venezuela. Email: danny_villegas1@yahoo.com

${ }^{2}$ Universidad Politécnica Territorial “JJ Montilla”, Guanaré, Venezuela. Email: danny_villegas1@yahoo.com

${ }^{3}$ Universidad Nacional Experimental de los Llanos Occidentales "Ezequiel Zamora", Guanaré, Venezuela. Email: danny_villegas1@yahoo.com

${ }^{4}$ Universidad Nacional de Jaén, Perú. Email: manuel.milla@unj.edu.pe
} 


\section{INTRODUCCIÓN}

El frijol (Vigna unguiculata) es una de las leguminosas de granos cultivadas con fines alimenticios desde épocas remotas, aun cuando no se ha determinado con certeza su lugar de origen. Sus granos contienen $23 \%$ de proteínas, $56 \%$ de carbohidratos, además de grasa, fibras solubles (pectinas), minerales y vitaminas (Graterol et al., 2006; De Gouveia et al. 2005). En la agricultura moderna, una de las especies vegetales alimenticias de gran demanda por sus cualidades nutricionales y que revisten gran interés comercial por su diversidad de consumo, características nutricionales e importancia ambiental económica son las leguminosas de grano, dentro de las cuales destaca el V. unguiculata (Alban 2012), que crece en las regiones tropicales y subtropicales del mundo Mena et al. (2015).

El cultivo de frijol $V$. unguiculata es una de las principales actividades de la economía campesina en varias regiones del país, de mucha importancia como generador de ingresos y empleo rural (21 jornales/ha), debido a que demanda bajos costos de producción, y como producto de la alimentación básica de la población vulnerable por ser fuente de proteína, calorías, fibra, minerales y vitaminas (Araméndiz et al. 2016). La agricultura es una actividad primaria básica para la subsistencia del hombre. En tal sentido, se requiere conocer los sistemas de producción agrícola, a fin de mejorar y obtener mayores rendimientos de los cultivos. En ese orden, la mayor parte de la superficie agrícola del país se explota bajo sistemas agrícolas tradicionales, por lo que se requiere conocer mejor y valorar las experiencias de los campesinos dedicados a la agricultura tradicional y en base a ello diseñar y proponer sistemas de producción más eficientes y sustentables.

Es importante validar y difundir información sobre los sistemas de producción intensificada e integrada y sobre opciones de diversificación, especialmente para los pequeños agricultores, incluyendo el uso eficiente del recurso hídrico, métodos de labranza cero y sistemas integrados. La mayoría de los productores de frijol son de pequeña escala, con escasos recursos que no les permite el uso de muchos insumos externos tales como; abono, plaguicidas o semilla mejorada, maquinaria y equipos, mano de obra, afectando la productividad del mismo. En tal sentido, la presente investigación tiene como objetivo principal clasificar y caracterizar pequeños sistemas de producción de frijol (V. unguiculata), en los municipios Guanare, Papelón y San Genaro de Boconoíto, del estado Portuguesa, permitiendo identificar los factores que inciden en la producción de este rubro.

\section{MATERIALES Y MÉTODOS \\ Área de estudio}

El área de estudio está ubicada al sur del estado Portuguesa, abarcando los municipios Guanare, Papelón y San Genaro de Boconoito. Estos tres municipios se encuentran en el área de influencia de la cuenca del río Guanare a una altura cercana a los $200 \mathrm{msnm}$, caracterizada por amplias llanuras 
conformadas por grandes sistemas de abanicos aluviales y planicies de desborde. La distribución de las precipitaciones muestra un incremento en dirección noroeste del área de estudio desde, aproximadamente 1.500-1.600 mm/anuales. En relación al área de estudio Ewel y Madriz (1968) destacan el bosque seco tropical (bs-T), zona de vida esta que incluye el bosque seco denso deciduo o semideciduo.

\section{Metodología}

Se utilizó información técnica, socioeconómica, agroecológica, e institucional de los registros de ASOGUANARE S.A. Las variables consideradas fueron: (1) Municipio, (2) Hectáreas sembradas, (3) Superficie total de la parcela, (4) Hectáreas efectivas, (5) Rendimiento por hectárea (Kg/ha), (6) Fecha de siembra, (7) Variedad utilizada, (8) Preparación del terreno, (9) Tipo de siembra, (10) Limitaciones por plagas, (11) Limitaciones por enfermedades, (12) Limitaciones por malezas, (13) Tipo de riego, (14) Limitaciones por equipo/maquinaria, (15) Limitaciones por cosechadoras, (16) Asistencia técnica, (17) Tipo de mano de obra. De las 17 variables seleccionadas se descartaron 8 variables, ya que para el total de productores (43), los mismos no reportaron variabilidad puesto que fueron caracteres comunes en cada una de las fincas.

Sobre la matriz de datos X, la cual está conformada por el total de vectores de las observaciones X[ij], $\mathrm{j}=1, \ldots, \mathrm{p}$ y donde los mismos representan la j-ésima variable para las observaciones. En este caso la matriz X está conformada por " $n$ " observaciones con "p" variables, es decir, 43 fincas (filas) x nueve variables seleccionadas (columnas). Las 43 fincas corresponden con el total de productores inscritos en ASOGUANARE S.A. Esta matriz fue analizada con la ayuda del entorno de programación R 3.4.4, el cual permitió realizar un análisis de componentes principales (ACP) basado en la matriz de correlación, para determinar: Valores propios y proporción de varianza explicada, matriz de vectores propios de la matriz de transformación, matriz de correlación entre las variables originales y los componentes principales, proyección de las unidades (fincas) en el plano de las dos primeras componentes $(\mathrm{CP} 1 * \mathrm{CP} 2)$.

El ACP es una técnica que transforma ciertas variables correlacionadas en otras incorrelacionadas, de media cero, que pueden escribirse como combinaciones lineales de las primeras y que se llaman componentes principales, éstas pueden ordenarse por la magnitud de su varianza, la cual está dada por un valor propio de la matriz.

Desde sus orígenes, el ACP tiene aplicación a ciertas tareas de diagnóstico o predicción y es una poderosa técnica multivariada que puede ser aplicada a un sin número de problemas en las ciencias. De este modo, el método ACP ha sido utilizado por climatólogos y meteorólogos para la delineación de patrones de temperatura, presión, precipitación, etc. (Richman, 1981; Legates, 1991; Brier y Meltesen, 1976; Davis et al., 1991); en investigaciones de clasificación de áreas homogéneas de suelos (Ovalles y Collins, 1988; Kosaki y Juo, 1989 y Nash y Daugherty, 1990); en estudios con insectos (Sharma, 1988); 
en análisis atmosféricos (Barnett, 1977 y Kutzbach, 1967); botánica (Ariyo y Odulaja, 1991), horticultura (Hobson et al, 1990 y Iezzoni y Pritts, 1991); fitopatología (Shuh et al., 1987); ecología (Pla, 1982; Morin et al., 1989); en clasificación de sistemas de producción de leche (Quevedo, 1991) y de fincas agropecuarias (Demey et al., 1994). En nuestro caso, el ACP se utilizó como herramienta para clasificación de sistemas de producción de $V$. unguiculata en los tres municipios con el fin de caracterizar los subgrupos homogéneos discriminados mediante el ACP.

\section{RESULTADOS Y DISCUSIÓN}

En la Tabla 1, se muestra una descripción estadística de las variables, donde se puede observar una alta variabilidad con coeficientes de variación que van desde $23,48 \%$ hasta 109,90\%. Esta alta variabilidad se puede explicar por la diversidad asociada al manejo de producción de Frijol en la zona. Así mismo, es importante resaltar que en promedio se tienen parcelas de 49,11 has con 27,93 has disponibles para la siembra y 27,17 has efectivas. Por otro lado, se observó un rendimiento promedio 554,88 $\mathrm{Kg} / \mathrm{ha}$. Estos resultados evidencian que la producción de frijol en la zona está representada por pequeños sistemas de producción, con una alta variabilidad en el rendimiento lo que coincide con lo reportado por Torres et al. (2013) en un estudio sobre la caracterización de la producción de frijol.

Tabla 1. Descripción estadística de las variables asociadas a 43 fincas y parcelas productoras de Frijol (Vigna unguiculata).

\begin{tabular}{lccc}
\hline \multirow{2}{*}{ Variable } & \multicolumn{3}{c}{ Estadística } \\
\cline { 2 - 4 } & Media $(\bar{X})$ & Desviación estándar $(\mathbf{S})$ & $\begin{array}{c}\text { Coeficiente de Variación } \\
(\%)\end{array}$ \\
\hline Superficie total (ha) & 49,11 & 50,63 & 103,10 \\
Hectáreas sembradas & 27,93 & 29,78 & 106,62 \\
Hectáreas efectivas & 27,17 & 29,86 & 109,90 \\
Rendimiento $(\mathrm{kg} / \mathrm{ha})$ & 554,88 & 130,29 & 23,48 \\
\hline
\end{tabular}

En la Tabla 2, se muestra la matriz de correlación, donde se observa que existe una correlación altamente significativa $(\mathrm{P}<0,01)$ entre las hectáreas sembradas y efectivas con la superficie total (ha), así mismo, existe una correlación altamente significativa $(\mathrm{P}<0,01)$ entre las hectáreas sembradas y las hectáreas efectivas, no obstante, el rendimiento no mostró alguna relación con las variables antes señaladas. Estas correlaciones encontradas en dicha matriz sugieren la existencia de algún tipo de asociación entre estos factores, lo que justifica la aplicación del análisis de componentes principales por el método de la matriz R. En ese orden, en la Tabla 3 se muestran los autovalores y las proporciones de varianza de la matriz de datos asociada a las fincas productoras de frijol en los tres municipios. Utilizando el 
criterio de incluir sólo aquellos valores que fuesen mayores a 1, se seleccionaron tres componentes principales, los cuales explican el $71,87 \%$ de la variación, la cual es una proporción significativa del total $(\geq 70 \%)$, lo que permite reducir la dimensión de la matriz y a su vez se simplifica considerablemente tanto la interpretación como el tratamiento posterior que haga de los mismos.

También puede observarse mediante el método gráfico que la primera "onda" incluye los primeros tres componentes y la segunda alcanza hasta el séptimo componente. La Figura 1 muestra la distribución de la varianza explicada entre los 9 componentes principales de los datos bajo análisis, coincidiendo este criterio de selección con el de Kaiser.

Tabla 2. Matriz de correlación de las variables asociadas a 43 fincas y parcelas productoras de Frijol (Vigna unguiculata).

\begin{tabular}{lcccc}
\hline \multirow{2}{*}{ Variable } & \multicolumn{3}{c}{ Coeficiente de Correlación y su significancia } \\
\cline { 2 - 5 } & $\begin{array}{c}\text { Superficie total } \\
\text { (ha) }\end{array}$ & $\begin{array}{c}\text { Hectáreas sem- } \\
\text { bradas }\end{array}$ & $\begin{array}{c}\text { Hectáreas } \\
\text { Efectivas }\end{array}$ & Rendimiento (kg/ha) \\
\hline Superficie total (ha) & & $0,522^{* *}$ & $0,528^{* *}$ & $0,275 \mathrm{~ns}$ \\
$\quad$ Hectáreas sembradas & & $0,998^{* *}$ & $0,166 \mathrm{~ns}$ \\
Hectáreas efectivas & & & $0,182 \mathrm{~ns}$ \\
Rendimiento (kg/ha) & & & \\
\hline
\end{tabular}

Tabla 3. Autovalores y proporción de varianza de la matriz de datos de las variables asociadas a 43 fincas y parcelas productoras de Frijol (Vigna unguiculata).

\begin{tabular}{cccc}
\hline Componente & Autovalores & Proporción de varianza \% & Varianza acumulada \% \\
\hline 1 & 3,043 & 33,806 & 33,806 \\
2 & 2,169 & 24,104 & 57,910 \\
3 & 1,257 & 13,964 & 71,875 \\
\hline
\end{tabular}

Los datos correspondientes a la nueva forma de agrupación de las fincas se presentan en la Figura 2, puede verse que el análisis discrimina tres categorías distintas de fincas o unidades de producción. De igual manera en la Tabla 5 se muestra una descripción de los grupos antes señalados, en donde se observan tres grupos (A, B y C) diferenciados fundamentalmente por la superficie de la finca y la variedad utilizada. 


\section{Scree Plot}

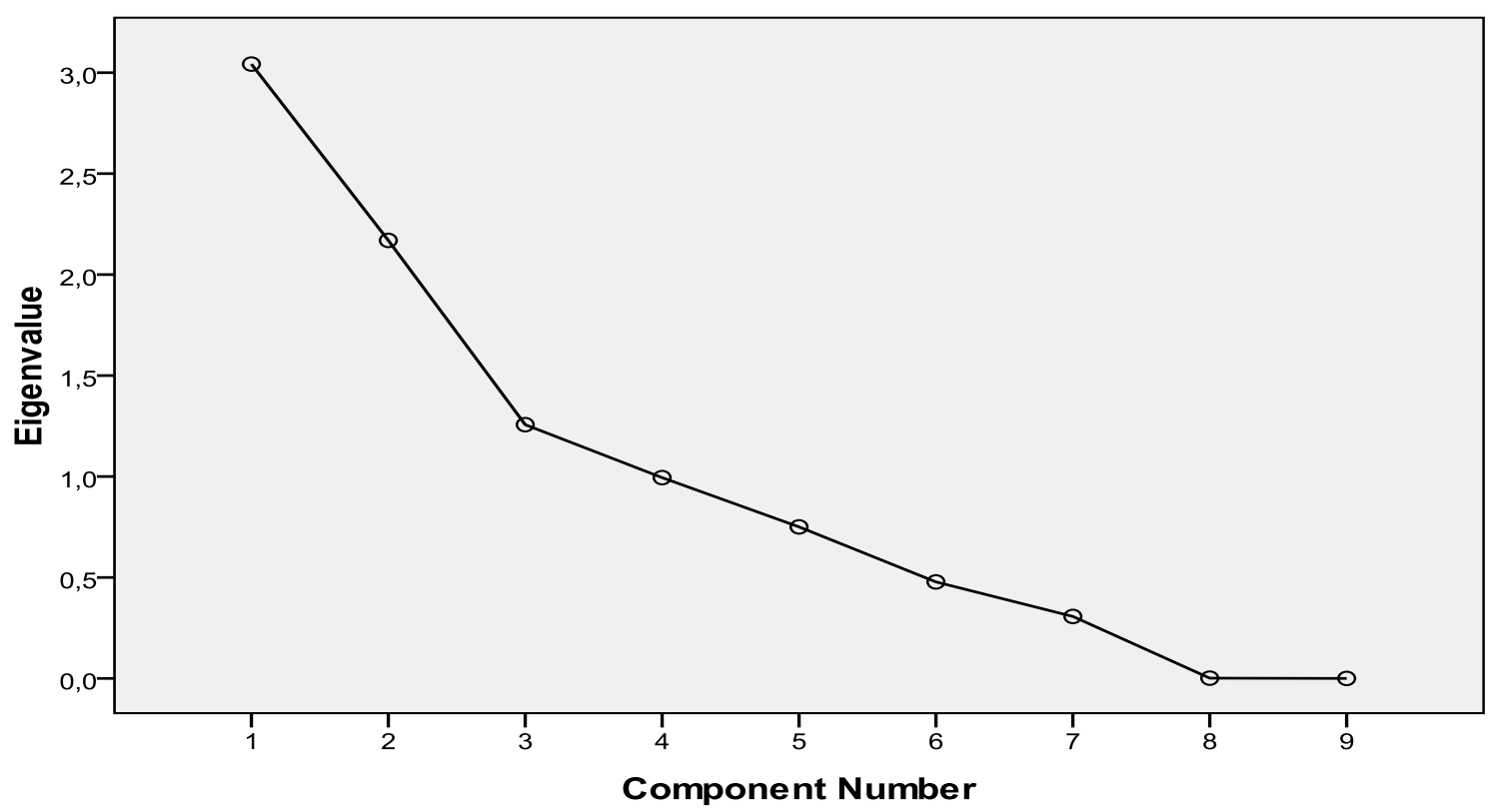

Figura 1. Sedimentación de los autovalores y componentes principales de la matriz de datos de las variables asociadas a 43 fincas y parcelas productoras de Frijol (Vigna unguiculata) en los municipios Guanare, Papelón y San Genaro de Boconoíto.

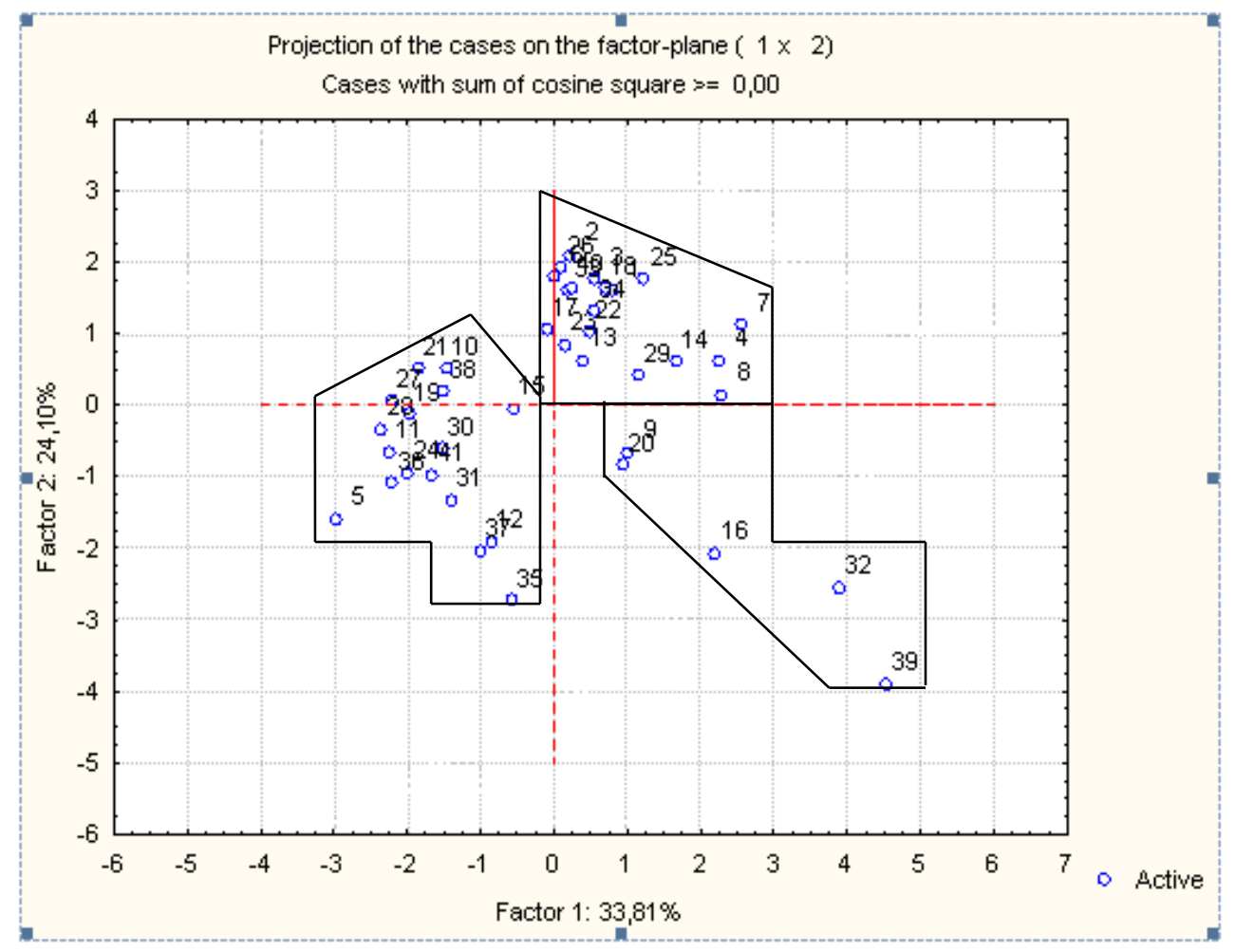

Figura 2. Proyección de las 43 fincas y parcelas en el plano de las dos primeras componentes (CP1 * CP2). 
Los resultados indican que el primer componente es el que tiene la varianza más alta 33,80\% y por lo tanto mayor capacidad explicatoria de la variabilidad en los datos (Tabla 4), así mismo se observan valores positivos en proporciones más o menos análogas de aquellas variables que en su conjunto reflejan el tamaño, escala, dimensión o superficie de las 43 fincas y parcelas, entre ellas; superficie total (ha), superficie efectiva (ha) y superficie sembrada (ha), por otra parte, el segundo componente muestra una relación entre variables asociadas a limitaciones por cosecha y por mano de obra y fecha de siembra; además se observa el efecto negativo que producen estas limitaciones sobre la fecha de siembra. Estos resultados evidencian lo señalado por otros autores, entre ellos González et al. (2007), quienes señalan que en pequeños sistemas de producción la mano de obra empleada en la mayor parte del proceso productivo es la del productor y su grupo familiar. Esto indudablemente explica la relación y el subsecuente efecto entre los factores antes señalados, específicamente en la segunda componente.

Tabla 4. Análisis de componentes principales sobre la matriz rotada (varimax) de las variables asociadas a 43 fincas y parcelas productoras de Frijol (Vigna unguiculata).

\begin{tabular}{lccc}
\hline & \multicolumn{3}{c}{ Componente } \\
& $\mathbf{1}$ & $\mathbf{2}$ & $\mathbf{3}$ \\
\hline Municipio & 0,007 & $-0,112$ & 0,844 \\
Superficie total (ha) & 0,713 & $-0,211$ & 0,285 \\
Hectáreas sembradas & 0,932 & $-0,113$ & $-0,184$ \\
Hectáreas efectiva & 0,938 & $-0,100$ & $-0,182$ \\
Fecha de siembra & $-0,238$ & $-0,625$ & 0,600 \\
Rendimiento (kg/ha) & 0,399 & 0,106 & 0,286 \\
Limitaciones por equipo y maquinaria & $-0,204$ & 0,942 & $-0,061$ \\
Variedad utilizada & $-0,063$ & $-0,425$ & $-0,402$ \\
Limitaciones por mano de obra & $-0,204$ & 0,942 & $-0,061$ \\
\hline
\end{tabular}

Tabla 5. Clasificación y caracterización de 43 fincas y parcelas productoras de Frijol (Vigna unguiculata) en los municipios Guanare, Papelón y San Genaro.

\begin{tabular}{cccccccc}
\hline & \multicolumn{4}{c}{ Promedio } & \multicolumn{2}{c}{ Variedad utilizada (\%) } \\
\cline { 2 - 8 } Grupo & $\begin{array}{c}\text { Superficie } \\
\text { total (ha) }\end{array}$ & Hectáreas sembradas & $\begin{array}{c}\text { Hectáreas } \\
\text { efectivas }\end{array}$ & Rendimiento (kg/ha) & Bayo & BPN & Bayo/BPN \\
\hline A (12\%) & 91 & 86 & 85 & 630 & 60 & 0 & 40 \\
B (43\%) & 56,23 & 21,53 & 20,58 & 550 & 42,1 & 36,8 & 21,1 \\
C (45\%) & 28,85 & 18 & 17,53 & 538,24 & 64,7 & 29,4 & 5,9 \\
\hline
\end{tabular}


En la Tabla 6, se observa la clasificación y caracterización del manejo del cultivo de Frijol (V. unguiculata) en los municipios Guanare, Papelón y San Genaro de Boconoíto, donde se muestra que los productores del grupo A y B iniciaron el periodo de siembra en el mes de noviembre el primero y noviembre-diciembre para el segundo, respectivamente, así mismo se puede observar que ambos grupos no se presentaron limitaciones por equipo y maquinaria y la mano de obra empleada fue propia. Finalmente, los productores del grupo $\mathrm{C}$ se caracterizaron por iniciar la siembra en el período de octubre a diciembre, presentando la mayoría de estos $(94,1 \%)$ limitaciones por equipo y maquinaria, así como también por mano de obra, estando conformado este último grupo por pequeñas parcelas. Estos resultados sugieren que la producción de frijol en estos tres municipios es de subsistencia con bajos niveles de uso de tecnología, lo que coincide con lo reportado por otros autores, entre ellos Torres et al. (2013) en un estudio sobre la caracterización de la producción de frijol.

Tabla 6. Clasificación y caracterización del manejo del cultivo de Frijol (Vigna unguiculata) en 43 fincas y parcelas de los municipio Guanare, Papelón y San Genaro de Boconoíto.

\begin{tabular}{|c|c|c|c|c|c|c|}
\hline \multirow[b]{2}{*}{ Grupo } & \multicolumn{4}{|c|}{$\begin{array}{l}\text { Fecha de siembra } \\
\text { (\% fincas/grupo) }\end{array}$} & \multirow{2}{*}{$\begin{array}{c}\text { Limitaciones } \\
\text { por equipo y maquinaria } \\
(\%)\end{array}$} & \multirow{2}{*}{$\begin{array}{c}\text { Limitaciones } \\
\text { por } \\
\text { mano de obra } \\
(\%)\end{array}$} \\
\hline & $\begin{array}{c}01-15 \\
\text { oct }\end{array}$ & $\begin{array}{c}01-15 \\
\text { nov }\end{array}$ & $\begin{array}{c}16-30 \\
\text { nov }\end{array}$ & $\begin{array}{c}01-15 \\
\text { dic }\end{array}$ & & \\
\hline A & 0 & 60 & 40 & 0 & 0 & 0 \\
\hline B & 0 & 0 & 21,1 & 78,9 & 0 & 0 \\
\hline $\mathrm{C}$ & 5,9 & 52,9 & 29,4 & 11,8 & 94,1 & 94,1 \\
\hline
\end{tabular}

\section{CONCLUSIONES}

El análisis de componentes principales (ACP) permitió identificar seis factores que están relacionados con la producción de frijol en 43 fincas o parcelas en los municipios Guanare, Papelón y San Genaro de Boconoíto. El ACP permitió clasificar las 43 fincas y parcelas en tres grupos de productores discriminados principalmente por la dimensión o tamaño de la finca o parcela. Se observó un grupo conformado por fincas de mayor tamaño (91 has), en su mayoría dedicadas a la siembra de frijol como principal actividad productiva del sistema y rendimientos de $630 \mathrm{Kg} / \mathrm{ha}$. Los otros dos grupos reportaron rendimientos inferiores $(550 \mathrm{Kg} / \mathrm{ha}$ y $538,24 \mathrm{Kg} / \mathrm{ha})$, respectivamente, diferenciándose del primer grupo en cuanto a superficie total, ya que en ellos se ubican fincas con un promedio de 56,26 has y 28,85 has, cuya actividad principal no es la producción de frijol, sino que producen otros rubros o están dedicados 
la actividad pecuaria. Finalmente, la variedad de frijol más común en los tres grupos es Bayo, la cual es sembrada entre un $40 \%$ y $60 \%$ en las fincas y parcelas de los tres municipios.

\section{REFERENCIAS BIBLIOGRÁFICAS}

Albán, M. 2012. Manual de cultivo de Frijol caupí. caupí (V. unguiculata) 7.10. 15.17pp.

Araméndiz, H., Cardona, C. y Combatt, E. 2016. Contenido Nutricional de Líneas de Fríjol Caupí (Vigna unguiculata L. Walp.) Seleccionadas de una Población Crollalla.http://www.scielo.cl/scielo.php?script=sci_arttext\&pid=S0718-07642016000200007. Inf. Tecnológica 27(2):53-60.

Ariyo, O. and A. Odulaja. 1991. Numerical analysis of variation among accessions of okra Abelmoschus esculentus (L) Moench. Ann. Bot. 67:527-531.

Barnett, T.P. 1977. The principal time and space scale of the pacific trade wind fields. J. Atm. Sci. 34:221-236.

Brier, G. W. and T.G. MELTESEN. 1976. Eigenvector analysis for prediction of time series. Notes and Correspondence 15:1307-1312.

Chatfield, C. and A.J. Collins. 1980. Introduction to multivariate Analysis. Chapman and Hall. New York. $246 \mathrm{p}$.

Davis, M.J., F. Estis, P. Bloomfield and J.F. Monahan. 1991. Complex principal component analysis of sea-level pressure over the eastern USA. International J. Clim. 11:27-54.

De Gouveia, M., Bolivar, A., Lopez, M., Salih, A. y Perez H. 2005. Participacion de agricultores en la seleccion de materiales geneticos de frijol (Vigna unguiculata) evaluados en suelos ácidos de la Parroquia Espino, estado Guarico (Venezuela). Cuadernos de Desarrollo Rural 54:113-130.

Demey, J., Adams, M. y Freites, H. 1994. Uso del Método de Análisis de ComponentesPrincipales para la Caracterización de Fincas Agropecuarias. Agronomía Tropical 44(3): 475-497.

Ewell, J. y Madriz, A. 1968. Zonas de vida de Venezuela. Memoria explicativa sobre el mapa ecológico. Ministerio de Agricultura y Cría; Dir. Investigación. Caracas. 265 p

González, B., Torres, E., Giunta, I., Muñoz, J., Díaz, G. 2007. Respuestas Campesinas frente a la apertura comercial: Evaluación situacional de la agricultura a nivel de pequeños productores en la zona central de la Costa ecuatoriana. Los Ríos Ecuador. 112p. 
Graterol, I., Gonzalez, R., Avila, J; De la Cruz, R; Lopez, A; Velasquez, L; Almeida; N y Pieruzzin, N. 2006. Abono foliar en variedades de frijol y epocas de aplicacion del glifosato en siembra directa del frijol 'Pico negro' en el estado Portuguesa. INIA Divulga Vol. 9: 30-33.

Hobson, G., C. Gough and C. Townley. 1990. Measuring consumer reaction to the flavour of fresh tomatoes. Acta Hortic. 259:107-116.

Iezzoni, A.F. and M.P. Pritts. 1991. Applications of principal component analysis to horticultural research, Hort. Sci. 26:334-338.

Kosaki, T. and A. Juo. 1989. Multivariate approach to grouping soils in small fields. I. Extraction of factors causing variation by principal component analysis. Soil Sc. Plant Nutr. 35:469-477.

Kutzbach, E.J. 1967. Empirical eigenvectors of sea-level pressure, surface temperature and precipitation complexs over North America. J. Appl. Meteo., 6:791-802.

Legates, D. 1991. The effect of domain shape on principal components analysis. Inter. J. Clim. 11:135146.

Mena, H.K.; Krishna, K.R. and Singh, B. 2015. Character associations between seed yield and its components traits in cowpea [Vigna unguiculata (L.) Walp.]. Indian J. Agric. Res., 49 (6): 567-570.

Morin E., A. Bouchard and P. Jutras. 1989. Ecological analysis of disturbed riverbanks in the Montreal area of Québec. Env. Manag. 13:215-225.

Nash, M.H. and L.A. Daugherty. 1990. Statistical comparison of soil map-unit boundaries. Soil Sci. Soc. Am. J. 54:1677-1681.

Ovalles, F.A. and M.E. Collins. 1988. Variability of Nortwest Florida soils by principal component analysis. Soil Sci. Soc. Am.J. 52:1430-1435.

Pla, L. E. 1982. Un modelo en el análisis de la vegetación del estado Falcón. Tesis Magister Scientiarum. Universidad Central de Venezuela UCV. Postgrado de Estadística. Venezuela. 245 p.

Pla, L. E. 1986. Análisis multivariado: método de componentes principales. OEA. 97 p.

Quevedo, R.L. 1991. Una metodología para un estudio de las fincas de producción lechera en el estado Yaracuy. Universidad Central de Venezuela, UCV. Facultad de Agronomía. MaracayVenezuela. 340 p.

Richman, B.M. 1981. Obliquely totated principal components: an improved meteorological map typing technique. American Meteorological Society 20:1145-1159.

Sharma, H. 1988. No-choice cage technique to screem for resistance to sorghum midge (Dip- 
tera:Cecidomyiidae). J. Econ. Entomol. 81(1):415-422.

Shuh, W., M. Jeger and R. Frederisksen. 1987. The influence of soil environment of the incidence of sorghum downy mildew: a principal component analysis. Phytopathology. 77(2):128-131.

Torres, E., Caiza, D., Sánchez, A., Reyes, M., González, B., Torres, A., Cedeño, A., Haro, A. 2013. Caracterización de la producción de frijol en la provincia de Cotopaxi Ecuador: Caso Comuna Panyatug. Ciencia y Tecnología. 6(1): 23-31. 